\title{
Nikolaus von Myra in Verehrung und Brauch nördlich der Alpen
}

\author{
Peter Dinzelbacher \\ Universität Innsbruck \\ peter.dinzelbacher@aon.at \\ https://dx.doi.org/10.12795/futhark.2011.i06.04
}

\section{Zusammenfassung:}

In kompakter Übersicht wird mit vielen konkreten Beispielen gezeigt, wie Nikolaus von Myra auch nördlich der Alpen zu einem vielverehrten heiligen Patron (zunächst namentlich der Seeleute, dann der Kinder) wurde. Seine Gestalt wurde in der volksläufigen Devotion mit vorchristlichen Schreckfiguren assoziiert; Nikolaus und Krampus sanken jedoch bis in die Gegenwart zu rein folkloristischen Gestalten ab, die nur noch als Erziehungsinstanz für kleine Kinder fungieren.

\begin{abstract}
:
In a compact summary based on many concrete images this study demonstrates that Saint Nicholas of Myra turned into a much venerated saintly patron even north of the Alps (first for sailors, then for children). His figure was associated in popular devotion with a pre-Christian frightful figure, Krampus, but both then converted into purely folkloric characters that function only in the area of education for small children.
\end{abstract}

\section{Schlüsselbegriffe:}

Nikolaus, Heiligenverehrung, Kinder, Volkskunde, Brauchtum, Teufel

\section{Keywords:}

Saint Nicholas, devotion of saints, children, popular culture, customs, devil 


\section{Verehrung}

Sowohl die kirchliche als auch die volksläufige Verehrung ${ }^{1}$ des anatolischen Bischofs und Heiligen, dessen Lebensdaten uneinheitlich zwischen 270 und 365 angesetzt werden, entwickelte sich nördlich der Alpen wesentlich langsamer als in Italien. Die Verbringung seiner Gebeine aus dem Osten nach Bari 1087 - das Epochenjahr der Nikolausdevotion in Europa - erfolgte ja eben deswegen, da dort bereits ein lebhafter Kult bestand, wie auch in anderen mediterranen Städten, nicht zuletzt in dem um die Reliquien konkurrierenden Venedig. Schließlich zeigte ein Siegel des Normannenherzogs Robert Guiskard, der Bari 1071 als den letzten byzantinischen Stützpunkt erobert hatte, schon zwei Jahre vor dem Reliquientransfer den hl. Nikolaus ${ }^{2}$.

Nach Norden, wo Nikolaus erst 818 zum ersten Mal (in Fulda) erwähnt wird $^{3}$, verbreitete sich die Kunde von diesem besonders mächtigen Thaumaturgen auf verschiedenen Wegen. Einerseits von Byzanz aus, wobei namentlich die oströmische Prinzessin Theophanu, die 972 die Gemahlin des späteren Kaisers Otto II. (973 - 983) und Mutter Otto III. (983 - 1002) wurde, eine Vermittlerrolle spielte. Durch sie wurde Nikolaus zum „Hausheiligen” der deutschen Königsdynastie der Ottonen und Patron vieler von diesen gestifteten Kirchen ${ }^{4}$.

Eine weitere Route nach dem Norden führte von Rom aus, wo eine griechische Kolonie existierte, die als Vermittlerin anzusehen ist; einige der zahlreichen Pilger aus dem Norden werden auch mit Nikolaus bekannt geworden sein. Andererseits führte die Verbindung der süditalienischen Normannen mit ihren Verwandten in der Normandie zur raschen Verbreitung des Kultes in Westfrankreich.

Aber erst die Entführung der Reliquien nach Bari brachte die Nikolaus-Verehrung auch nördlich der Alpen richtig in Schwung, zumal

1. Zur Heiligenverehrung vgl. grundsätzlich Dinzelbacher/Bauer pass. und Dinzelbacher, Handbuch 335-342 und die dort zitierte Literatur; zuletzt Ders., Lebenswelten des Mittelalters, Badenweiler 2010, $511 \mathrm{ff}$.

2.Metken 22.

3. Metken 18. Daß Liudger, Gründerbischof des Bistums Münster, noch vor 800 in Billerbeck eine Kirche dem heiligen Nikolaus geweiht habe, erscheint nicht gesichert.

4. G. Wolf, Kaiserin Theophanu, die Ottonen und die Beginn der St. Nikolaus-Verehrung in Mitteleuropa, in: Kaiserin Theophanu, Prinzessin aus der Fremde, Köln 1991, 27-38. 
viele Kreuzfahrer die Seereise nach dem Heiligen Land von Bari aus unternahmen. Er wurde zum einzigen orientalischen Bischof, den auch die lateinische Kirche feiert. Da das Hauptpatronat des Heiligen der Schutz der Seefahrer war, entstanden besonders in den Hafenstädten Nikolauskirchen und konnte der Heilige zum Stadtpatron avancieren wie etwa in Amsterdam. Bald wurde sein Kult von Norddeutschland durch die Hanse auch in Skandinavien eingeführt, wo als einer der frühesten Träger seines Namens der dänische König Niels (reg. 1104-1134) gilt. In Ungarn zählte sein Fest schon 1092 zu den Hauptfesten des Landes 5 .

Im späten Mittelalter vervielfachten sich in ganz Europa, besonders in den Küstengebieten, die Nikolauskirchen und -kapellen, allein England z.B. soll vor der Reformation ca. $400 \mathrm{inm}$ geweihte Kirche besessen haben ${ }^{6}$. Die Verbindung des Heiligen mit dem Jüngsten Gericht scheint besonders in Skandinavien vollzogen worden zu sein, wo Nikolaus statt des Erzengels als Seelenwäger fungiert ${ }^{7}$ und auch auf Kalkmalerein am Ende der Zeiten direkt neben der Madonna steht, um seine Verehrer zu schützen (z.B. in Birkerød, DK, um 1340) ${ }^{8}$.

Wie für so viele andere Heiligenkulte war auch hier die Übertragung von Nikolaus-Reliquien von höchster Bedeutung. So entstand durch einen Pilger, der Ende des 11. Jahrhunderts ein Fingerglied des Bischofs aus Bari mitgebracht hatte, am Ufer des Flusses Meurthe bei Nancy in Lothringen Saint-Nicolas-de-Port, bald ein von weither aufgesuchter und auch im Hochadel beliebter Wallfahrtsort ${ }^{9}$. Vor allem bat man den Heiligen hier um Befreiung aus Gefangenschaft, und nach einem Bericht von 1464 sollen damals so viele als Votivgabe hinterlegte Ketten in der Kirche gewesen sein, dass man sie mit 50 Wagen nicht hätte fortbringen können. Auch Strafwallfahrten als kirchliche oder weltliche Busse wurden häufig nach Saint-Nicolas befohlen. „Monseigneur Saint Nicholas" avancierte zum Patron Lothringens, als 1477 Karl der Kühne in der Nähe des Ortes vernichtend

5. A. Binterim, Die vorzüglichsten Denkwürdigkeiten der Christ-Katholischen Kirche V/1, Mainz 1829, 514.

6. D. H. Farmer, The Oxford Dictionary of Saints, Oxford 2. Aufl. 1987, 316.

7 Gad 290.

8. L. Liepe, Den medeltida kroppen, Lund 2003, 180-182.

9. M. Parisse, in LexMA VII, cc. 1186-7. 
geschlagen werden konnte. Erst der Dreißigjährige Krieg und die Pestepidemien der Zeit beendeten den Ruf dieses Pilgerzentrums ${ }^{10}$.

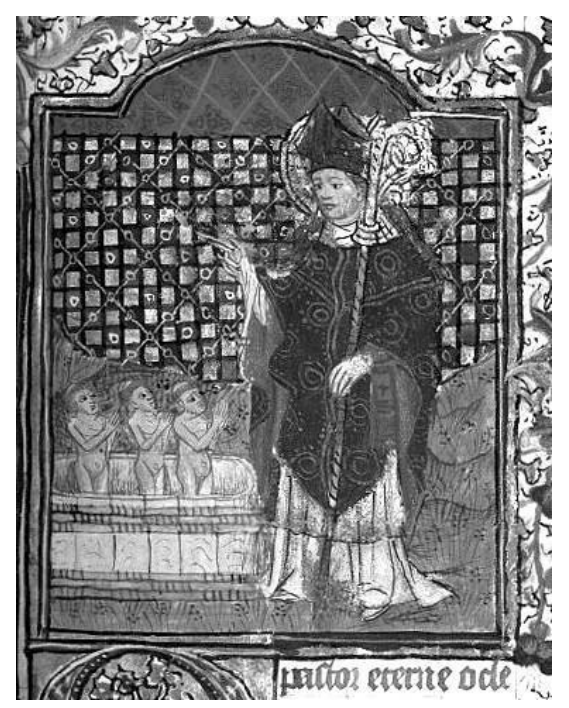

Abb. 1 Der hl. Nikolaus erweckt die toten Kleriker. Miniatur aus dem Stundenbuch des Jean de Vy, vor 1450 (Metz, Bibliothèques)

Wie die lateinische Liturgie des Heiligen verbreiteten sich auch seine Legenden überall auf dem Kontinent, wobei dem Anglonormannen Robert Wace mit seiner 1562 gereimte Verse umfassenden Vie de saint Nicolas (vor 1155) der zeitliche Primat innerhalb der volkssprachlichen Literaturen zukommt ${ }^{11}$. Zentrale Bedeutung bekam natürlich, wie auch sonst für die Verbreitung der Viten und der Ikonographie der Heiligen, die Legenda Aurea des Jakobus von Varazze, von der im 14. und 15. Jahrhundert auch zahlreiche Versionen in allen Volkssprachen

10. Metken 26-30; Saint-Nicolas, Actes pass.

11. La vie de saint Nicolas, par Wace, ed. E. Ronsjö, Lund 1942. 
kursierten. Die Nikolaus-Legende dürfte in keiner von innen gefehlt haben, wie auch kaum ein einem anderen Legendar ${ }^{12}$.

Analog dazu entstanden unzählige bildliche Darstellungen des Heiligen, die sich rasch von dem Modell der byzantinischen Ikonenmalerei lösten und stattdessen die Standardikonographie für bischöfliche Heilige mit den persönlichen Attributen (drei Kugeln, drei Kinder, Schiff) adaptierten. Die goldenen Kugeln hatte der Heilige Jungfrauen zugeworfen, um ihnen ein artiges Leben zu ermöglichen, die Kinder bzw. Kleriker hatte er nach einer ungerechten Hinrichtung wieder auferweckt, das Schiff war durch seine Hilfe vor dem Kentern bewahrt worden ${ }^{13}$. Diese Ikonographie war so verfestigt, daß sie sogar der Satan mit Hilfsteufeln nachahmte, wenn er sich zur Täuschung einer Visionärin in den Thaumaturgen verwandeln wollte ${ }^{14}$. Dazu traten die sonstigen Szenen seiner Vita. Einblattdrucke, Wachsmodeln, Bilderbogen, Hinterglasbilder, Keramikmalerei u.dgl.m. machten das Bild des Nikolaus weiter volksläufig, ebenso Bildsäulen und andere Kleindenkmale.

12. W. Williams-Krapp, 'Nikolaus', in: Die deutsche Literatur des Mittelalters. Verfasserlexikon VI, 2. Auf. 1987, cc. 1037-9.

13. Mit dem Wasser blieb dieser Heilige auch sonst verbunden, so wurde seine Statue verwendet, um Hochwasser aufzuhalten (M. Cormack, The Saints in Iceland, Bruxelles 1994, 64).

14. Entre dieu et Satan. Les visions d'Ermine de Reims ( $\left.{ }^{*} 1396\right)$, ed. C. Arnaud-Gillet, Tavarnuzze 1997, $124 \mathrm{ff}$. 
Abb. 2 Typische spätbarocke Darstellung des Heiligen mit seinem häufigsten Symbol, den goldenen Kugeln (Mühlbach am Hochkönig/Salzburg, Pfarrkirche)

Unter den religiösen Schauspielen ist das kurze lateinische des Hilarius von Orléans (+ M. 12. Jh.) vielleicht das älteste bekannte; dieser Ludus super iconia s. Nicolai behandelt in einer Mischung aus Latein und Altfranzösisch die Legende vom Bild des Heiligen, das auf die Schätze eines „Barbaren“ aufpassen soll, und endet mit der Bekehrung des Heiden ${ }^{15}$. Die Geschichte war beliebt, in Frankreich wurde sie u.a. im

15. Hilarii Aurelianensis versus et ludi, epistolae, ludus Danielis Belouacensis. Herausgegeben von W. Bulst u. M. Bulst-Thiele, Leiden 1989, 43-46. 
volkssprachigen Jeu de St Nicolas lebhaft auf die Bühne gebracht ${ }^{16}$; in extenso hat man sie u.a. in der Gewölbemalerei von St. Maria Lyskirchen in Köln dargestellt (um 1240; statt des Barbaren wird hier ein Jude bekehrt); sie scheint der Grund dafür zu sein, dass der Heilige auch zum Patron der Diebe wurde ${ }^{17}$. Obwohl Strafwunder von fast allen Heiligen erzählt wurden, fällt doch auf, wie häufig dies der Fall bei Nikolaus war ${ }^{18}$, d.h. seine Spezialisierung auf die Rute geht jedenfalls ins Mittelalter zurück. Auch in Deutschland entstanden schon seit dem Hochmittelalter dramatische Bearbeitungen der Nikolaus-Wunder ${ }^{19}$. In der Gegenreformation kam es im Jesuitentheater zu einem neuen Schub hagiographischer Dramen, deren volkssprachige Versionen dann auch Einfluß auf die nicht wenigen Volksschauspiele gewannen, die vom 18. bis 20. Jahrhundert den Nikolaus der Legende popularisierten ${ }^{20}$. Die Zahl der volkssprachlichen Nikolausspiele, die jedenfalls bis weit ins 19. Jahrhundert hinein im katholischen Europa sehr beliebt waren, ist groß. Sie wurden unter kirchlicher Aufsicht gespielt und dienten primär der Vermittlung kirchlicher Normen, aber vermischt mit volkstümlicher Unterhaltung, wozu auch dem frommen Thema eigentlich ganz fremde Szenen eingebaut wurden, z.B. Streitgespräche, Schäferspiele, Schimmelbeschlagen, Totentanz ${ }^{21}$. Ansätze zu solcher komödienhafter Gestaltung finden sich freilich schon deutlich im Jeu de saint Nicolas des Arraser Dichters Jean Bodel $(+1210)$, der eine Variante desselben Themas auf Französisch bearbeitete, wie Hilarius auf Latein ${ }^{22}$.

Die Beliebtheit des Heiligen zeigt sich deutlich an den wachsenden Nachbenennungen; immer mehr Kinder wurden im hohen Mittelalter auf den Namen Nikolaus getauft und somit seinem Schutz unterstellt ${ }^{23}$. Im

16W. Tydeman ed., The Medieval European Stage 500-1550, Cambridge 3. Auf. 2008, 160

17. Wrede c. 1089.

18.Z.B. C. C. Coulton, Medieval Panorama, Cambridge 1945, 600.

19. K. Young, The Drama of the Medieval Church, Oxford 1933, II, 312-336.

20. Cf. z.B.: I. Greinz, Nikolaus-Volksschauspiele in Österreich, Diss. Innsbruck 1934; H. Schuhladen, Die Nikolausspiele des Alpenraums, Innsbruck 1984.

21. Viele Beispiele bei Schnell, pass.; A. Haberlandt, Taschenwörterbuch der Volkskunde Österreichs II, Wien 1959, 78-9.

22. Jean Bodel, Le Jeu de saint Nicolas, ed. Jean Dufournet, Paris 2005.

23. M. Mitterauer, Ahnen und Heilige. Namengebung in der europäischen Geschichte, München 1993, 280-1, 340-43. 
13. Jahrhundert war er in der Gegend um Basel bereits der vierte unter den beliebtesten Namen, im 14. und 15. Jahrhundert stand er in vielen norddeutschen Regionen als "Claus" an erster Stelle. Eine Reihe von Familiennamen wie z.B. engl. Nixon, Nicholsen, dt. Classen etc. leitet sich vom Vornamen Nikolaus ab.

Anders als bei der orthodoxen Dynastie der Romanows hat aber keine der großen westlichen Herrscherfamilien Nikolaus zum Leitnamen gemacht.

Nach dem Tridentinum verlief auch die offiziell-kirchliche Nikolausverehrung in den nunmehr weniger individuellen Bahnen der disziplinierten Catholica. Bemerkenswert ist, dass Nikolaus, anders als andere volkstümliche Heilige - man denke an Franziskus oder Maria Magdalena - in der Literatur und Musik der Moderne wohl nur mit einem wirklich hervorragenden Werk Eingang gefunden hat, nämlich der Kantate „Saint Nicolas“ von Benjamin Britten (1948).

\section{Brauch}

Mehr als wohl fast alle anderen europäischen Volksbräuche, die mit einem Heiligen verbunden sind, existierte und existiert das mit Nikolaus verbundene in einer enormen regionalen und zeitlichen Vielfalt, von der nur ein paar Beispiele erwähnt werden können. „Der Wechsel der Gestalt (unter dem gleichen Namen) und des Namens (bei gleicher Gestalt) ist im Nikolausbrauch hundertfach belegt und erschwert eine Übersicht." ${ }^{24}$ Bescherung, Rüge und Umgang bilden jedenfalls die wesentlichen, immer wieder auftretenden Komponenten.

Der ältere Brauch, Schüler am Tag ihres Patrons zu beschenken, wurde natürlich auch weiter ausgeübt, nachdem Nikolaus im 12. und 13. Jahrhundert zum bevorzugten Heiligen dieser Gruppe geworden war, womit die bis heute feste Verbindung des Nikolaus mit der Kinderbescherung fixiert war. Der Nikolausabend entspricht somit dem Epiphanietag in Italien, wo Befana die Geschenke bringt. Religionsgeschichtlich betrachtet, trat der Heilige damit in die Funktion einer vorchristlichen Glaubensgestalt ein, in den lateinischen Quellen

24. Beitl, K. (Hg.), Wörterbuch der deutschen Volkskunde, Stuttgart 3. Aufl. 1974, 601. 
Diana oder Abundantia genannt, die, unsichtbar allerdings, die Häuser besuchten und Gaben mitbrachte ${ }^{25}$.

Wie Kinder im 15. Jahrhundert am Nikolaustag eine Gabe erwarteten, geht etwa aus folgendem frühneuhochdeutschen Gebet hervor, in dem um ein Geschenk gebeten wird, das in ein Schiffsmodell gelegt werden soll (wie später dann in Schuhe und Socken):

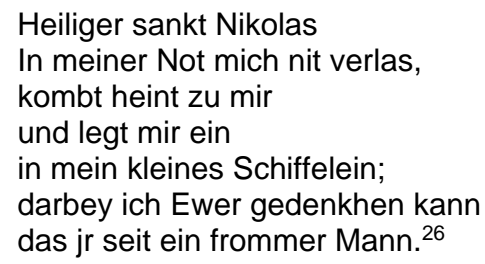

Die ausführliche Polemik der Reformatoren gegen den Nikolausbrauch und ihr Bemühen, den bischöflichen Gabenbringer durch das Christkind zu ersetzen, zeigt, dass er um 1500 schon fest verwurzelt war; er lässt sich dann in dieser Funktion kontinuierlich bis in die Gegenwart verfolgen. Der Übergang vom Nikolausgeschenk zum Weihnachtsgeschenk ist sogar bei Luther direkt fassbar: Während er 1535 für Kinder und Gesinde noch Geschenke zu „S. Niclas“ einkaufte, sprach er zehn Jahre später von der Christbescherung. In einer deutschen Sprichwortsammlung von 1548 werden gleichwertig Christ oder Nikolaus als Gabenbringer genannt ${ }^{27}$. Bald sollte letzterer jedoch in den reformierten Territorien ganz vom Christkind verdrängt werden, da man in Nikolaus einen unerwünschten Rest der katholischen Heiligenverehrung sah.

Vielfach spann man aus den Heiligenlegenden Patronate heraus, so auch bei Nikolaus: Dass er zum Helfer für heiratslustige Mädchen wurde, erklärt sich daraus, daß er nach der Legende drei Schwestern mit einer Mitgift ausgestattet hatte. So sind aus Süddeutschland und Nordfrankreich Nikolaussteine bezeugt, deren Bewegung oder

25. Janssen 154-157.

26. Metken 55.

27. Meisen 26. 
Erkletterung eine baldige Hochzeit garantieren sollte ${ }^{28}$, auf Fünen schmückten zum selben Zweck die Mädchen eine Statue des Heiligen ${ }^{29}$, und in der Normandie sangen die sie:

Patron des filles, Saint Nicolas,

Mariez-nous, ne tardez pas! ${ }^{30}$

Sein Patronat für Schüler, und dann Kinder überhaupt, ist ebenfalls aus einer Episode seiner Legende, nämlich der Errettung der drei ermordeten Jungen, zu erklären. Im Volksglauben und -brauch wurde dieses Patronat aber dann auch auf Kinderwunsch ausgedehnt: Der Zisterzienser Caesarius von Heisterbach berichtet um 1230, dass man im Kloster Burtscheid bei Aachen eine aus Griechenland importierte Ikone des Heiligen besaß, die Gebärenden zur Erleichterung ins Zimmer gehängt wurde ${ }^{31}$. In der Bretagne rieben sich Frauen mit einer Nikolausfigur den Leib, in manchen Alpenregion erzählte man den Kindern, Nikolaus bringe die Babies, die er von Bäumen pflücke, was sonst Aufgabe des Storchs ist ${ }^{32}$.

In Nordfrankreich wurde Nikolaus im Hochmittelalter zum Patron der Schüler und Studenten, vermutlich in Folge des entsprechenden Wunders in seiner Lebensbeschreibung wie auch seines Reisepatronats. An seinem Festtag wurden ein Mirakelspiel und eine Prozession veranstaltet. Doch wurden am 6. Dezember auch Feste gefeiert, die der kirchlichen Kontrolle zu entgleiten drohten. Denn dieser Termin zog im Frankreich des 13. und 14. Jahrhunderts das früher am Tag der Unschuldigen Kinder (28. Dezember) gefeierte Narrenfest an sich, das mancherorts von einem Knabenbischof regiert wurde. Diesen nannte man daher auch Nikolausbischof. Da das Schüler- und Kinderfest mit Geschenken an diese verbunden war, die teilweise in einem Heischegang eingeholt wurden, ist hier der Ursprung des Nikolaus als

28. Metken 32-33.

29. Gad 291.

30. Schnell VI, 121.

31. Caesarii Heisterbacensis monachi O. Cist. dialogus miraculorum 8, 76, rec. J. Strange, Coloniae 1851, II, 144-145.

32. Metken 39, 41. 
brauchtümlicher Gabenbringer zu sehen. ${ }^{33}$ Bis ins 18. Jahrhundert lässt sich an vielen Schulen dieser Knabenbischof nachweisen, bis ins 20. Jahrhundert gibt es noch vereinzelte Beispiele. Dies, wiewohl die mit dem Narrenfest einhergehenden Freizügigkeiten der Jugendlichen immer wieder mit Verboten der geistlichen und weltlichen Obrigkeiten belegt worden waren und natürlich auch aufklärerischer Kritik verfielen.

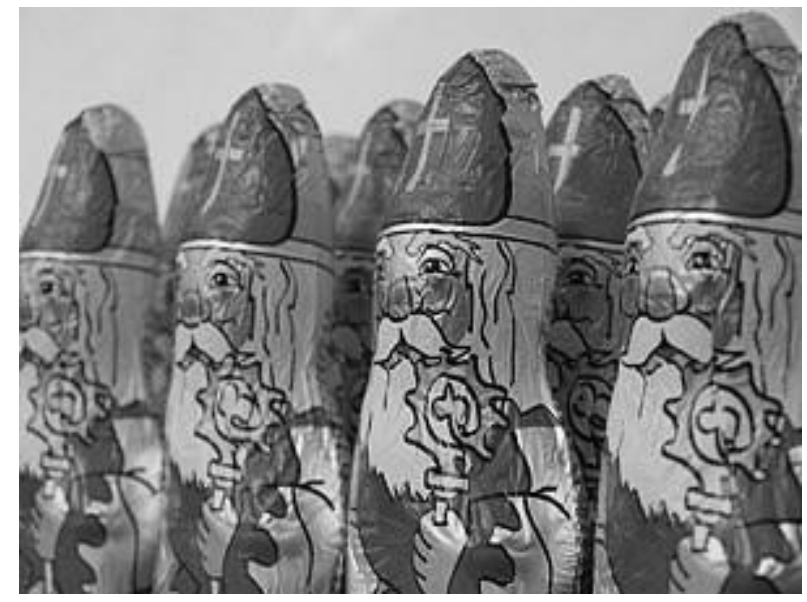

Abb. 3 Nikolaus als kommerzielles Produkt aus Schockolade

Üblich war und ist es wenigstens seit der Frühneuzeit, dass der Nikolaus in die Häuser kommt und die Kinder über inr religiöses und schulisches Wissen befragt. Früher kontrollierte er das sog. Klausenhölzchen, in dem eingekerbt war, wie viele Vaterunser ein Kind seit Martini gebetet hatte ${ }^{34}$. Tadel mit der Rute und Belohnung mit Geschenken folgte. Unter den Gaben, früher vor allem Nahrungsmittel

33. Metken 51.

34. K. Beitl, Das Klausenholz. Untersuchung der Gebetszählhölzer im vorweihnachtlichen Kinderbrauch, in Rheinisches Jahrbuch für Volkskunde 20, 1971, 7-92. 
und Spielzeug, heute auch Geld, sind besonders typisch Gebildbrote, also in Figurenform Gebackenes. Üblicherweise waren dies Menschenfiguren, die den Heiligen selbst meinten, aber auch Tierfiguren und unbelebte Objekte. Typischerweise wurden solche Figuren aus Lebkuchenteig gebacken, die bisweilen von beachtlicher Größe waren. Im 20. Jahrhundert wurde dieses Geschenk mehr und mehr durch industriell in entsprechende Formen gegossene und in bedruckte Goldfolie gewickelte Schokolade ersetzt. Daneben schenkte man den Kindern auch Krampusse in der Form von Pflaumenmännern, d.h. aus diesem Dörrobst zusammengesteckte Figuren ${ }^{35}$.

Die Atmosphäre des Nikolausabends, die ein deutscher Autor um 1880 beschrieb, hat auch der österreichische Verfasser dieser Zeilen aus seiner Jugend in den Fünfzigerjahren gut in Erinnerung: „Nach Eintritt der Dunkelheit wird die Wanderung angetreten und verkündet das Gerassel der Kette schon von weitem den in der halberleuchteten Stube teils ängstlich, teils freudig erregt harrenden Kindern die Ankunft des St. Nicolaus und Klaubauf [eine Teufelsfigur]. Die Stubentüre springt auf, majestätisch tritt St. Nicolaus herein, hinter inm der gefürchtete Klaubauf. Während die Kleinsten ängstlich auf den Armen der Mutter oder hinter ihrer Schürze Schutz suchen, flüchten die größeren hinter den Tisch, auf die Bank und bereiten sich beklommen Herzens auf das bevorstehende Examen vor..."36

Lohn und Strafe, die die beiden Figuren verteilen, erweisen sie psychologisch als ,Extensionen' der Eltern (die in der Regel diesen Besuch organisieren). Ihre Funktion war es, die elterliche Gewalt zu unterstreichen und zu stabilisieren, also die gültigen sozialen Normen durch Figuren zu befestigen, die von den Kindern halb oder ganz als über- bzw. untermenschliche Wesen geglaubt wurden und denen nicht die Vertrautheit der Eltern anhaftete ${ }^{37}$.

Bisweilen straft Nikolaus selbst mit der Rute; wahrscheinlich kam der Heilige zu diesem Attribut als Patron der Schüler, gebrauchten doch die Lehrer des Mittelalters dieses Erziehungsmittel so regelmäßig, dass

35.H. Ploß, B. Renz, Das Kind in Brauch und Sitte der Völker II, Leipzig 3. Aufl. 1912, 334 ff.

36 Schnell III, 10.

37. Vgl. Eberspächer $176 \mathrm{ff}$; $214 \mathrm{ff}$. 
sie in der bildenden Kunst innen nicht nur stets als Erkennungszeichen beigegeben wird, sondern auch der personifiziertren Grammatik ${ }^{38}$. Allerdings scheint es keine kirchlichen Darstellungen des Heiligen mit diesem Attribut zu geben. Für 1555 ist die Rute auch als „Geschenk“ des Nikolaus neben Nüssen, Äpfeln, Birnen, Kleidung... bezeugt, die von der Mutter für die Kleinen vorbereitet und dann als Gaben des nicht selbst auftretenden Heiligen gedeutet wurden ${ }^{39}$. Es konnte diese Strafrute sogar als Lebenszweig verstanden werden, gibt es doch viele Bräuche, bei denen "frisch und gesund Schlagen" keine Schmerzzufügung bedeutet, sondern eine Übertragung der Naturkraft der Pflanze auf den Menschen ${ }^{40}$.

Gelegentlich trat der Bischof auch beritten auf, sei es auf Esel, Pferd oder Bock, und fast immer in verkleideter Begleitung. Ursprünglich stellte diese nur die heranwachsende männliche Jugend; selten sind weibliche Begleiterinnen belegt, so die Nikolofrau bzw. das Klausenweiblein. Sporadisch und lokal gab es auch schon im 19. Jahrhundert weibliche Begleitpersonen, so eine weiß gekleidete Magd in Ratibor, Schlesien ${ }^{41}$. Mit den feministischen Strömungen innerhalb der katholischen Kirche, die zur Zulassungen von Ministrantinnen führten, kam es auch zu kirchlicherseits organisierten Begleiterinnen des Bischofs, die seine Engel spielen durften ${ }^{42}$. Sehr selten treten weibliche Heilige mit dem Nikolaus auf, und das nur wegen des Termins ihres Festes (Barbara am 4. Dezember, Luzia am 13. Dezember) ${ }^{43}$. Es scheint, dass gelegentlich auch eine eigenständige Gestalt, die Percht, in den Nikolaus-Umkreis gezogen wurde, die in dämonischen und freundlichen Varianten vorkam ${ }^{44}$.

Ein besonders spektakulärer Brauch zu Nikolaus, charakteristisch für die Alpenregionen, aber auch andere Teile Europas, sind Umzugsbräuche, bei denen er selbst die Hauptfigur sein kann, aber keineswegs sein muß. Weder lässt sich die Entstehung der

38. LCl II, c. 707.

39. Metken 57.

40. Vgl. Beitl (wie Anm. \#) 50-3.

41. Schnell I, 66.

42. Koenig Abb. 24.

43. Metken 66.

44. E. Timm, Frau Holle, Frau Percht und verwandte Gestalten, Stuttgart 2003. 
Umgangsbräuche genauer datieren oder lokalisieren, noch angeben, wann die dunklen Begleiter zur Bischofsgestalt dazukamen (oder vice versa $^{45}$. Sie heißen Teufel, Mann-Düvel, Beelzebock, Leutfresser, Bartl, Schwarze, Klaubauf, Krampus, Pluto, Stämpes usw. ${ }^{46}$ Ihre typischen Attribute sind die Rute, die Ketten, der Sack (in den unartige Kinder gesteckt werden sollen). Dabei wird die Kette wohl ein umgedeuteter Rest aus der Legende vom Descensus (Abstieg) Christi in die Vorhölle darstellen, wo er Satan in Fesseln gelegt habe. Natürlich gibt es eine große, den lokalen Gegebenheiten entsprechende Variationsbreite, im nördlichen Skandinavien z.B. trug der Begleiter des Bischofs RenntierHaut und drohte, die Kinder in die eisigen Berge mitzunehmen und bei Wölfen und Bären auszusetzen ${ }^{47}$. Eine deutlich gezähmte Version stellt der Knecht Ruprecht vor, der sich aus Thüringen in vielen Teilen Deutschlands verbreitete. Er war in einen roten Kapuzenmantel gekleidet und konnte den Bischof auch ganz ersetzen. Diese Kleidung und die Zipfelmütze sind die Kennzeichen des säkularisierten Nikolaus bzw. Santa Claus, wie er mit größter Breitenwirkung v.a. durch das von dem Arzt Heinrich Hoffmann selbst gezeichnete Bilderbuch "Struwwelpeter" (1845 und zahllose Neuauflagen und Übersetzungen bis in die Gegenwart) verbreitet wurde und $\mathrm{zu}$ dessen Ahnen vielleicht die Personifikation der Winters in Plastik und Malerei gehört. Die Umwandlung des Heiligen bzw. der zwiespältigen Brauchtumsfigur in den nur mehr lieben Weihnachtsmann begann schon mit der Kritik am „Nikolausgespenst“ durch die Aufklärer des späten 18. Jahrhunderts, die sich auch gegen die mit dieser Figur verbundene Prügelpädagogik wandten ${ }^{48}$. Man kann das Resultat als "Idyllisierung" und Verbürgerlichung dieser Gestalt bezeichnen ${ }^{49}$.

Die älteste eindeutige Nachricht vom teuflischen Begleiter des Nikolaus scheint aus 1420 zu datieren: Für damals sind aus Basel Dämonenmasken bezeugt, die den Kinderbischöfen als Diener beigegeben waren; innen wurde das Betreten der Kirchen und das

45. Meisen hat beides versucht, doch keineswegs schlüssig, sondern rein hypothetisch. 46. Meisen 418; Metken 64.

47. Schnell VI, 12.

48. Eberspächer $93 \mathrm{ff}$.

49. Ebd. 117. 
Laufen während der Messen untersagt ${ }^{50}$. Man muß jedoch davon ausgehen, dass zu der Zeit, wo ein Brauch für uns das erstemal in einer Quelle fassbar wird, diese keineswegs seine tatsächlich älteste Form widerspiegeln muß. Wie kam Nikolaus in diese Gesellschaft? Aus seiner Legende, wo er Böse tatsächlich mehrfach auftritt? Es gibt aber doch so gut wie keine Heiligenvita, in der der Teufel nicht vorkäme, um den Gottesmann oder die Gottesfrau zu versuchen und doch unterliegen muß. Auch der siegreiche Kampf des Heiligen um eine Seele noch in diesem Leben oder schon im Jenseits ist der Topos vom hilfreichen Patron, der die Seinen beschützt, und keineswegs ein Spezifikum der Nikolaustradition. Vielmehr ist davon auszugehen, dass hier andere Figuren aus dem Mitwinterbrauchtum mit dem Teufel der Kirchenlehre verschmolzen wurden.

In manchen Gegenden wurde Nikolaus selbst sogar zu einer oft unheimlichen Figur umgedeutet: Rumpel- oder Bullerklas, Hellenklos, Kloaskerl... Auch im Äußern erinnert bisweilen nichts mehr an den Kirchenfürsten, sondern er trat im dunklen Mantel auf. Am weitesten getrieben wurde diese Entwicklung wohl in Schlesien, wo als Gabenbringer der Bartel mit Bärenmaske und Strohkleid zu den Kindern $k^{51}{ }^{51}$. „Der Geschenkespender N., der vielerorts mit Absicht in alte, schlechte Kleider gehüllt ist, Pelzwerk tragen muß, in Stroh gewickelt ist oder dessen Beine wenigstens mit Strohseilen oder gewundenen Weidenruten gegürtet sind, der Ketten trägt, gehört eindeutig dem Kreis der in den Wintermonaten umziehenden Masken und Gestalten an."52 So die Einschätzung eines der romantischen bis nationalistischen ,Volkstumsideologie' zweifellos unverdächtigen Folkloristen. Bemerkenswerter Weise kommt die Kurzform des Namens des Heiligen sogar in manchen deutschen Schimpfnamen vor: Filznickel (Geizhals), Bosnigl (boshafter Kerl), Schweinigel (< Schweinennickel)... ${ }^{53}$

Die als Rudel u.a. im Alpenland auftretenden Kläuse erinnern nur dem Namen nach den den mediterranen Heiligen; sie sind in Erscheinung und Verhalten Perchtengestalten, also dämonische Figuren, welche mit Peitschengeknall und Glockengetöse die bösen

50. Meisen 434.

51. Metken 60, 67.

52. M. Zender, in: Meisen XI.

53. W. Pfeifer (Hg.), Etymologisches Wörterbuch des Deutschen, München 1995, 923. 
Geister verjagen wollen. Solche vermummte Gestalten treten an anderen Terminen ganz ohne Zusammenhang mit einem Heiligen auf, u. zw. besonders in der dunkelsten Zeit des Jahres, den Raunächten ${ }^{54}$. Der Eigenname Nikolaus wurde hier letztlich aufgrund der zeitlichen Überschneidung so sehr Appellativum, dass er sogar für Masken am Heiligen Abend, zu Silvester und in der Fastnacht gebraucht wurde ${ }^{55}$

Beim nächtlichen „Klausjagen“ in der Nordschweiz (etwa Küssnacht) wurden zwei Nikolausdarsteller von einer Schar von "Clausjägern" begleitet. Sie springen auf der Straße vor, drehen sich um sich selbst, springen wieder zurück, machen eine Kniebeuge, vollführen also ritualisierte, tanzartige Bewegungen. Ihr Gefolge macht mit Kuhglocken, Hörnern usw. einen möglichst lauten Lärm ${ }^{56}$. Im oberösterreichischen Salzkammergut wurde und wird der mit weißem Priestergewand, Mitra und Stab ausgestattete Nikolaus von einer Horde von mit Bock- und Ziegenfellen gekleideten Schreckmasken begleitet, die besonders die jungen Mädchen mit Ruß schwärzen, sich auch gern in Raufereien mit jungen Männern - „Zivilisten“ - verwickeln ließen und lassen. Unzählige Variationen haben sich bei diesen Gestalten entwickelt; die Schwarzen Klasen in Amsterdam z.B., die nach schlimmen Kindern fahnden ${ }^{57}$, sind wiederum den kirchlichen Teufeln ähnlicher usw. Man hat hier vonseiten der Verhaltensforschung sicherlich zutreffend darauf hingewiesen, dass das Verlarven den Burschen eine durch den Brauch gedeckte Möglichkeit bot, unerkannt in körperlichen Kontakt mit dem anderen Geschlecht zu kommen, und dass die Glocken der Maskenträger nicht nur der Unheilabwehr dienen, sondern auch einem Imponiergehabe entsprechen. So war und ist die Rolle des Krampus bei den Burschen auch viel beliebter als die des frommen Bischofs ${ }^{58}$. Aus den westfriesischen Inseln ist schon aus dem Beginn des 20. Jahrhunderts bezeugt, dass beide Geschlechter an dem Treiben der "Sunderklazen" beteiligt waren; ein uneheliches Kind nannte man dort "'n lutje potje van Sunterkloas" 59 .

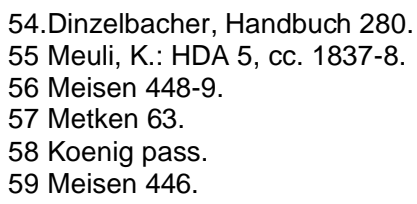




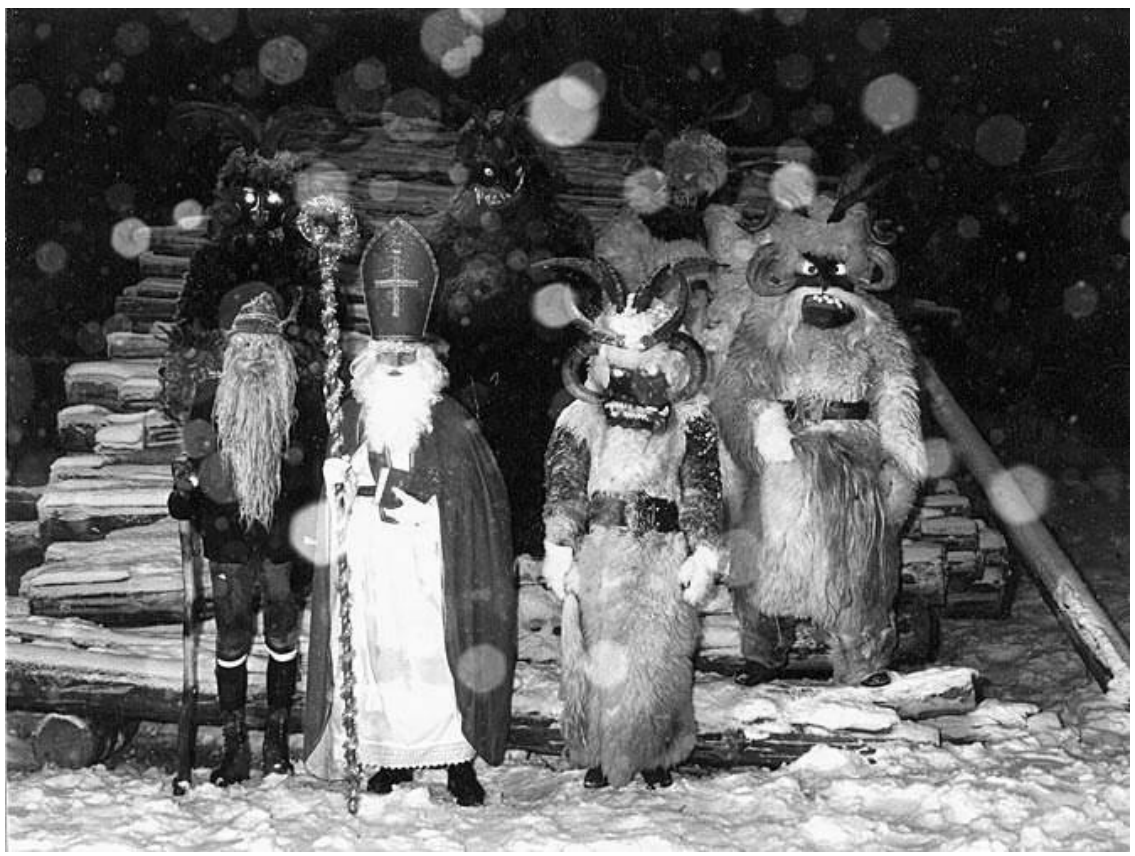

Abb. 4 Nikolausumzug mit Krampussen (Großarl/Salzburg)

Ob man im Mittelalter in diesen Horden wiederkehrende Seelen oder Dämonen sah, wird schon damals je nach Ort und Epoche verschieden gewesen sein. Aber es ist sehr wahrscheinlich, dass die KrampusScharen in den rituellen Darstellungen der Wilden Heers der Seelen mindestens genauso ihre Ahnen hatten wie in den Teufeln des religiösen Schauspiels, und auch die Verteilung der Gaben könnte eine Tradition des vorchristlichen Wintersonnwendfestes weiterführen ${ }^{60}$; auch die seit der Jahrtausendwende bezeugten Wilden Waldleute in ihren

60 Während die ältere deutsche Volkskunde die erstgenannte Tradtionslinie überbetonte, möchte sie die jüngere mit Meisen ganz leugnen, beide Male weniger aus wissenschaftlichen Gründen denn aus ideologischen Vorurteilen. 
Fellgewändern dürften in diese Ahnengalerie gehören. Daß die Toten zum Jahreswechsel die Lebenden besuchen, ist ja eine weithin im Indoeuropäischen belegte Erscheinung61. Ob auch die seltene Vorstellung, Nikolaus sei der Herr über das Fegefeuer, in diesen Zusammenhang gehört, wie in der Vision des englischen Bauern Thurkill $(1206)^{62}$ oder einer dänischen Predigt des 15. Jahrhunderts ${ }^{63}$ beschrieben, bleibe dahingestellt.

\section{Folklore und Folklorismus}

Mischung traditioneller Motive mit aus ganz anderem Umfeld entnommenen Elementen und primär kommerzielle Funktion kennzeichnet die Umgestaltung wenn nicht Auflösung des Nikolausbrauches der Gegenwart. Dieser ist oft vereinsmäßig organisiert und soll Touristen anziehen; allemal ist er in seinen pittoreskesten Ausformungen ein Medienereignis. Die Modernisierung ist vielfach überdeutlich: In Amsterdam etwa wird Sinterklaas feierlich von einem Schiff in die Stadt geholt, er reitet auf einem Schimmel, wird aber von einer Band Mohren auf Motorrollern - manchmal kommen sie auch per Fallschirm - sowie Indianern und Kamelen begleitet. Hier hat die Kommune die Sache in die Hand genommen, denn der Heilige wird feierlich vom Bürgermeister empfangen usw. ${ }^{64}$

Im 19. Jahrhundert hat die Figur des Weihnachtsmannes den traditionellen Heiligen zuerst speziell in den protestantischen Gebieten Deutschlands, dann weithin, verdrängt ${ }^{65}$. Die heute im Dezember in der typischen rot-weißen Kutte vor den meisten städtischen Kaufhäusern stehenden Weihnachtsmänner oder Santas oder Pères Noel sind sehr entfernte und völlig säkularisierte Verwandte des Nikolaus, die vom amerikanischen Santa Claus abstammen und $\mathrm{zu}$ einer reinen Reklamefigur mutierten. Als (bezahlter) Besucher von Krankenhäusern

61 Janssen 81-87.

62 P. Dinzelbacher, Himmel, Hölle, Heilige. Visionen und Kunst im Mittelalter, Darmstadt 2002, 84.

63 A. Riising, Danmarks middelalderlige Praediken, Køpenhavn 1969, 305.

64 Metken 71.

65 Eberspächer pas. 
oder Altersheimen usw. hat er eine karitativ-unterhaltende Funktion erhalten.

Aus der vormals reichen Zahl von Liedern und Gedichten zum Nikolaus ist heute im deutschen Sprachraum eigentlich nur mehr ein besonders anspruchsloses allgemein bekannt:

„Laßt uns froh und munter sein,

Und uns heut' von Herzen freu'n!

Lustig, lustig, trallerlallera,

Nun ist Niklas-Abend da!“

Die Verkleidung als Krampus, in der mancher (sonst strafwürdige) Aggressionsabbau erlaubt ist und wo man eigene Ängste abreagieren kann, ist bei Burschen immer noch beliebt, und noch dominiert der Usus, dass der Brauch des Krampuslaufens ganz primär von Angehörigen dieser Altersgruppe durchgeführt wird; doch findet man in der ersten Dezemberwoche auch schon Schulkinder in solchen Kostümen. 


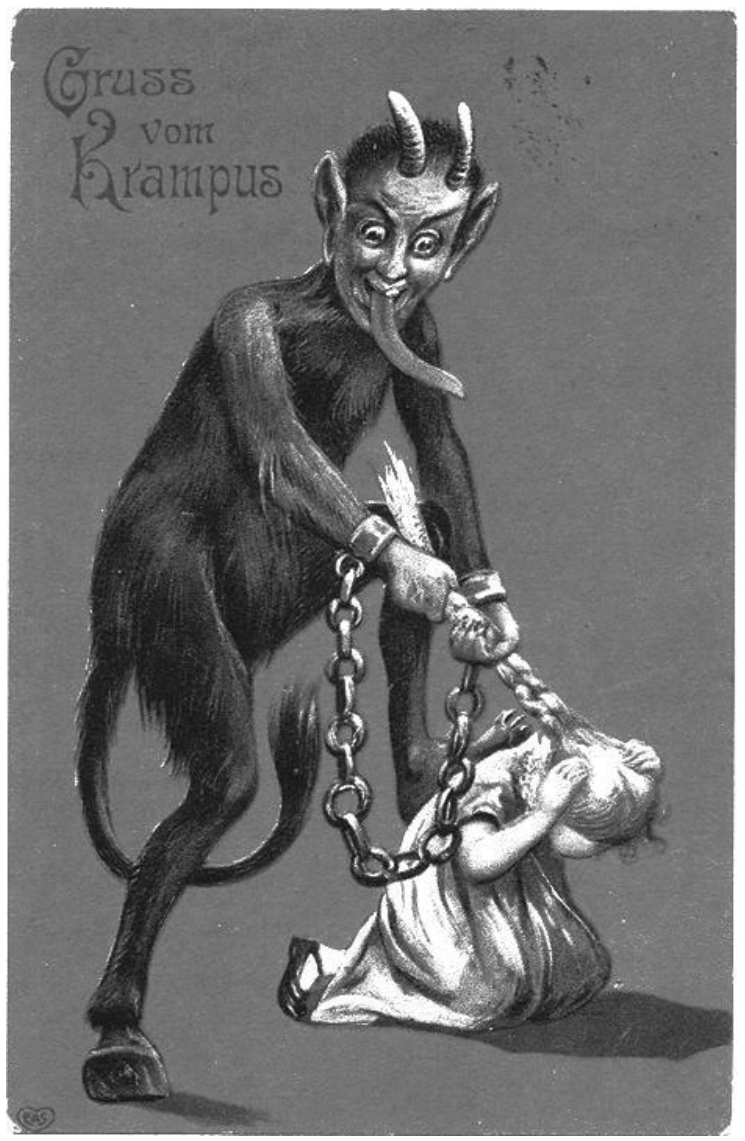

Abb. 5 Krampus straft Kind, Grußpostkarte der Firma EAS, Mitte 20. Jh.

Erwachsene gehören dagegen äußerst selten zu den Verlarvten (so im niederländischen Amerland) ${ }^{66}$. Da sich bei diesem Brauch an einem größeren Ort mehrere entsprechende Gruppen bilden, kann es vorkommen, dass ein Haus am Festabend mehrere Male heimgesucht

66 Janssen 47-50. 
wird und sich begegnenden Maskengruppen in ritualisierter Form begrüßen oder bekämpfen ${ }^{67}$.

Die früheren Masken waren formal deutlich aus dem spätmittelalterlichen Schauspiel entlehnt, wo so oft Dämonen in entsprechenden Kostümen auf der Bühne zu sehen waren und auch die umliegenden Straßen verunsicherten ${ }^{68}$. In letzter Zeit zeigen sie nicht ganz selten einen Formenwandel: Die Larven gleichen nun Schreckgestalten aus der Science Fiction und Phantasy-Filmwelt. In Salzburg und Tirol kann sich ein Tod (oder mehrere Totenkopfmasken) in die Gruppe der Klaubaufs mischen; dort spielen auch gelegentlich die Kinder den Maskenzug der Jugendlichen nach, wobei dann auch Mädchen Nikoläuse sein können ${ }^{69}$. Aber schließlich hat man den Nikolaus auch schon in Begleitung des Schneewittchens und der Sieben Zwerge gesehen (Remiremont, $1965)^{70}$. Auch für gläubige Katholiken ist heute der Nikolaus bestenfalls eine Brauchtumsfigur, und gebetet wird zu ihm, anders als in Süditalien und in der Orthodoxie, nicht mehr ${ }^{71}$.

\section{Bibliographie}

M. Becker-Huberti, Der Heilige Nikolaus. Leben, Legenden und Bräuche, Köln 2005

P. Dinzelbacher, Handbuch der Religionsgeschichte im deutschsprachigen Raum II: Hoch- und Spätmittelalter, Paderborn 2000

P. Dinzelbacher, Mentalität und Religiosität des Mittelalters, Klagenfurt 2003

P. Dinzelbacher/D. Bauer (Hgg.), Heiligenverehrung in Geschichte und Gegenwart, Ostfildern 1990

\section{Koenig 23.}

68 Janssen 138, 148.

69 Koenig 20-21.

70 Metken 70.

71Eine kürzere Fassung dieses Beitrags mit anderer Bebilderung wurde im Ausstellungsbegleitband San Nicola publiziert. 
A. Döring (Hg.), Faszination Nikolaus. Kult, Brauch und Kommerz, Essen 2001

A. de Groot, Saint Nicholas. A psychoanalytic study of his history and myth, The Hague, Paris 1965

M. Eberspächer, Der Weihnachtsmann. Zur Entstehung einer Bildtradition in Aufklärung und Romantik, Stuttgart 2002

T. Gad, Nicolaus av Myra, in: Kulturhistorisk leksikon for nordisk middelalder 12, 1967, cc. 288-294

N. Hallinger, Der Nikolaus und seine Buttnmandl, Berchtesgaden 2004

Hoegaerts, J., De vriend van ieder kind? Sinterklaas en de katholieke huisvader in het negentiende-eeuwse Vlaanderen, in: Trajecta 17, 2008, 314-334

L. Janssen, Nicolaas, de duivel en de doden, Baarn 1993

O. Koenig, Klaubauf - Krampus - Nikolaus. Maskenbrauch in Tirol und Salzburg, Wien 1983

C. Mechin, Sankt Nikolaus. Feste und Brauchtum in Vergangenheit und Gegenwart, Saarbrücken 1982

K. Meisen, Nikolauskult und Nikolausbrauch im Abendlande, Düsseldorf 2. Aufl. 1981

S. Metken, Sankt Nikolaus in Kunst und Volksbrauch, Duisburg 1966

W. Mezger, Sankt Nikolaus. Zwischen Kult und Klamauk. Zur Entstehung, Entwicklung und Veränderung der Brauchformen um einen populären Heiligen, Ostfildern 1993 
Saint-Nicolas, Actes du Symposium de Juin 1985, Saint-Nicolas du Port 1988

San Nicola. Splendori d'arte d'Oriente e d'Occidente, ed. M. Bacci, Pesaro/Milano 2006

W. Scharrer, St. Nikolaus in Schwaben. Ein Volksheiliger in Kirche, Kunst und Brauchtum, Augsburg 1991

E. Schnell, Sanct Nicolaus, der heilige Bischof und Kinderfreund, sein Fest und seine Gaben, I-VI, Brünn 1883/86

K. Schunert, Vom Weltenrichter zum Weihnachtsmann. Neue Erkenntnisse zur Identität der Nikolaus-Brauchgestalt und zur Entstehung des Gabenbrauchs, Bremen 2005 [mir nicht zugänglich]

Wrede, A., Nikolaus, in Handwörterbuch des deutschen Aberglaubens [HDA] 6, cc. 1086-1107 